\section{HIV-1 over time: fitness loss or robustness gain?}

\author{
Morgane Rolland* , Christian Brander ${ }^{\ddagger}$, David C. Nickle*, \\ Joshua T. Herbeck*, Geoffrey S. Gottlieb§, Mary S. Campbells, \\ Brandon S. Maust* and James I. Mullins*§
}

In a recent issue of Nature Reviews Microbiolog $y^{1}$ Arien and colleagues discussed the possibility that HIV-1 is measurably attenuated over time, based on reduced replication fitness in vitro. Historical isolates out-competed recent isolates in 176 out of 238 comparisons $^{2}$, although only twice in 4 matched competitions between isolates of subtype B-CCR5. Whether decreased fitness in vitro equates with diminished HIV-1 virulence is debatable, especially without any evidence of reduced disease severity or transmission, as stable en $^{3-5}$ or worsening ${ }^{6,7}$ prognostic markers have been reported over time.

As an alternative to the possible adaptation to the host, we propose that HIV-1 is shifting towards the possession of increasingly robust population characteristics (providing greater resilience against niche perturbations), which sustain survival and proliferation during environmental fluctuations at the expense of replication fitness, so exemplifying the 'survival of the flattest' effect ${ }^{8}$.

HIV-1 evolution over time may correspond to the relocalization of circulating
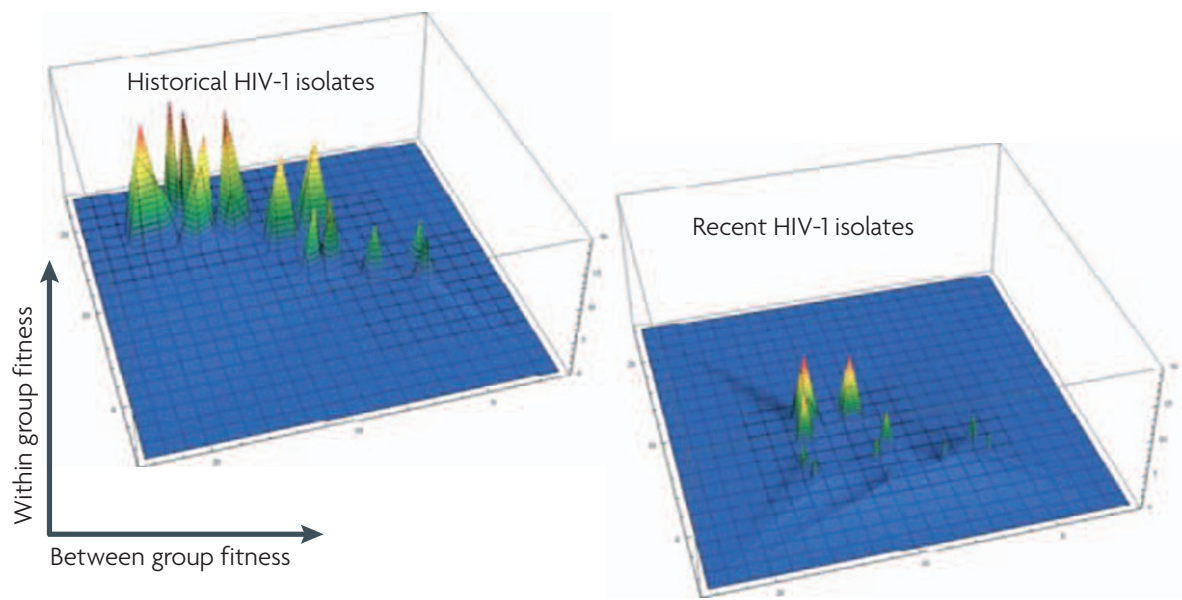

Figure 1 | Fitness landscapes of historical and recent isolates of HIV-1. The fitness landscapes shown represent data published by Arien and colleagues ${ }^{1}$. The height of the peaks corresponds to the mean replication fitness values for inter-group virus competitions, whereas the area under the peaks corresponds to the mean replication fitness values for intra-group competitions. Compared with historical isolates, recent isolates appear to constitute a network of lower fitness, with flatter peaks and fewer amplitude changes between peaks and valleys. 3D plots were obtained using Mathematica 6 (Wolfram Research, Champaign, Illinois, USA). perturbations. In silico studies have demonstrated that organisms on low, flat peaks can displace fast-replicating organisms on higher, narrower peaks - an effect termed 'survival of the flattest' ${ }^{8-11}$. This effect can also be viewed as a pressure to occupy highly connected, rather than sparse, areas of a network ${ }^{9}$. Although phenotypic constancy could appear to be at odds with the ever-increasing HIV-1 diversity, robustness and diversity are not mutually exclusive. Robustness combined with flexibility renders the exploratory behaviour of the virus less uncertain, by buffering it from lethal mutational effects ${ }^{12,13}$.

Although robust properties and escape mutations accruing in HIV-1 are plausible explanations for its lower fitness as the epidemic proceeds, we stress that diminished fitness does not necessarily imply attenuation. For individuals infected with evernewer HIV-1 generations, adverse outcomes could be expected. A partially debilitated virus could be better controlled by the host immune response, which would result in reduced or delayed disease progression and potentially attenuation. By contrast, a gain in robustness could ensure more transmissions and thus greater dissemination. Moreover, adaptation to common human leukocyte antigen alleles could gradually result in multi-escape variants, with impaired fitness balanced by a reduced susceptibility to cytotoxic T-lymphocyte recognition. This scenario of increased transmissions fostered by mutational robustness, coupled with less effective immune responses, contrasts starkly with attenuation.

* Department of Microbiology, University of Washington, Seattle, Washington 98195-8070, USA

${ }^{\ddagger}$ Partners AIDS Research Center, Massachusetts General Hospital, Harvard Medical School, Charlestown, Massachusetts 02192, USA.

${ }^{\S}$ Department of Medicine, University of Washington, Seattle, Washington 98195-8070, USA.

Correspondence to J.I.M. e-mail:jmullins@u.washington.edu

doi: 10.1038/nrmicro 1594-C1

Arien, K. K., Vanham, G. \& Arts, E. J. Is HIV-1 evolving to a less virulent form in humans? Nature Rev. Microbiol. 5, 141-151 (2007).

2. Arien, K. K. et al. Replicative fitness of historical and recent HIV- 1 isolates suggests HIV- 1 attenuation over time. AIDS 19, 1555-1564 (2005).

3. Holmberg, S. et al. Recent infection with human immunodeficiency virus and possible rapid loss of CD4 T lymphocytes. J. Acquir. Immune Defic. Syndr. Hum. Retrovirol. 9, 291-296 (1995).

4. Galai, N. et al. Temporal trends of initial CD4 cell counts following human immunodeficiency virus seroconversion in Italy, 1985-1992. The human immunodeficiency virus Italian seroconversion study. Am. J. Epidemiol. 143, 278-282 (1996).

5. Müller, V. et al. Stable virulence levels in the HIV epidemic of Switzerland over two decades. AIDS 20 889-894 (2006) 
6. Dorrucci, M., Rezza, G., Porter, K. \& Phillips, A. Temporal trends in postseroconversion CD4 cell count and HIV load: the concerted action on seroconversion to AIDS and death in Europe collaboration, 1985-2002. J. Infect. Dis. 195, 525-534 (2007).

7. Dorrucci, M., Phillips, A., Longo, B. \& Rezza, G Changes over time in post-seroconversion CD4 cell counts in the Italian HIV-seroconversion study: 1985-2002. AIDS 19, 331-335 (2005).

8. Wilke, C. O., Wang, J. L., Ofria, C., Lenski, R. E. \&
Adami, C. Evolution of digital organisms at high mutation rates leads to survival of the flattest. Nature 412, 331-333 (2001).

9. van Nimwegen, E., Crutchfield, J. P. \& Huynen, M. Neutral evolution of mutational robustness. Proc. Natl Acad. Sci. USA 96, 9716-9720 (1999).

10. Schuster, P. \& Swetina, J. Stationary mutant distributions and evolutionary optimization. Bull. Math. Biol. 50, 635-660 (1988).

11. White, J. S. \& Adami, C. Bifurcation into functional niches in adaptation. Artif. Life 10, 135-144 (2004).
12. Bloom, J. D., Labthavikul, S. T., Otey, C. R. \& Arnold, F. H. Protein stability promotes evolvability. Proc. Nat Acad. Sci. USA 103, 5869-5874 (2006).

13. Kirschner, M. \& Gerhart, J. Evolvability. Proc. NatI Acad. Sci. USA 95, 8420-8427 (1998).

\section{Acknowledgements}

This work was supported by grants from the Seattle Primary Infection Program (PO1 57005) and the University of Washington Center for AIDS Research (PO1 AI570005 and 61-480, RO1 Al058894 and Al047734). 